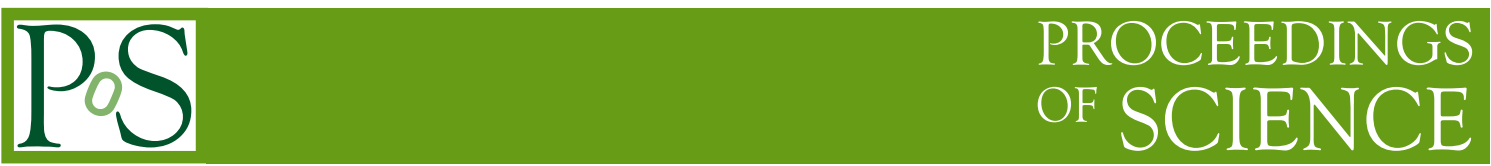

\title{
Parton Distribution Amplitudes and Non-Perturbative Renormalisation
}

\author{
P.A. Boyle ${ }^{a}$, D. Brömmel ${ }^{* b}$, M.A. Donnellan ${ }^{b, c}$, J.M. Flynn $^{b}$, A. Jüttner ${ }^{d}$ and C.T. \\ Sachrajda ${ }^{b}$ \\ ${ }^{a}$ School of Physics and Astronomy, University of Edinburgh, Edinburgh EH9 3JH, UK \\ ${ }^{b}$ School of Physics and Astronomy, University of Southampton, Southampton SO17 1BJ, UK \\ ${ }^{c}$ Deutsches Elektronen-Synchrotron DESY, 15738 Zeuthen, Germany \\ ${ }^{d}$ Institut für Kernphysik, Johannes Gutenberg-Universität Mainz, 55099 Mainz, Germany \\ E-mail: d.broemmelephys.soton.ac.uk
}

\section{RBC/UKQCD Collaborations}

We present results for the first two moments of the light-cone distribution amplitudes of the $\pi$ and $K$ pseudo-scalar mesons and of the $\rho, K^{*}$ and $\phi$ vector mesons. The calculations are performed on the RBC/UKQCD collaborations' ensembles generated with the Iwasaki gauge action and with $2+1$ flavours of domain wall fermions. In addition we also provide some results on the necessary non-perturbative renormalisation which we perform using the Rome-Southampton method. We discuss the benefits of the momentum source approach such as much smaller statistical errors and the possibility to see effects of the discretisation.

The XXVI International Symposium on Lattice Field Theory

July 14-19 2008

Williamsburg, Virginia, USA

\footnotetext{
*Speaker.
} 


\section{Introduction}

The first part of these proceedings will give an update on our results for the lowest moments of the leading twist meson distribution amplitudes (DAs) [1, 2]. Distribution amplitudes contain nonperturbative QCD effects that appear e.g. in hard exclusive processes but are universal hadronic properties and so do not depend on the process itself. They are important for form factors at large $q^{2}$ or $B$-decays and can be related to the Bethe-Salpeter wave function. Here we provide results for the pseudo-scalar DAs of $K$ and $\pi$ as well as for the vector DAs of $K^{*}, \rho$ and $\phi$. More details on these calculations by the RBC and UKQCD collaborations will be presented in an forthcoming paper.

The second part is devoted to the non-perturbative renormalisation of quark bilinears with and without derivatives. Renormalisation is necessary to obtain physical results for e.g. the matrix elements of moments of DAs or decay constants. We follow the Rome-Southampton method [3] with momentum sources [4] to calculate the renormalisation constants. The first is aimed at reducing uncertainties in the perturbative renormalisation while the latter is to improve upon the statistical errors from the standard point source approach.

The calculations are done on lattices with $24^{3} \times 64 \times 16$ and $16^{3} \times 32 \times 16$ points at a lattice spacing of $a^{-1}=1.729(28) \mathrm{GeV}$. The ensembles have been generated by the RBC/UKQCD collaborations using $N_{\mathrm{f}}=2+1$ domain wall fermions with an Iwasaki gauge action. Our light quark masses range from $a m_{\mathrm{q}}=0.005$ to 0.03 corresponding to pion masses from $331 \mathrm{MeV}$ to $672 \mathrm{MeV}$. The strange quark mass is kept fixed at $a m_{\mathrm{s}}=0.04$. Details on the ensembles have been reported in [5, 6].

\section{Meson distribution amplitudes}

The meson DAs are defined as non-local matrix elements on the light-cone. The leading twist pseudo-scalar and (longitudinal) vector DAs are

$$
\begin{aligned}
&\left.\left\langle 0\left|\bar{\psi}_{F_{1}}(z) \gamma_{\mu} \gamma_{5} \mathscr{U}(z,-z) \psi_{F_{2}}(-z)\right| \Pi^{+}(p)\right\rangle\right|_{z^{2}=0}=\mathrm{i} f_{\Pi} p_{\mu} \int_{-1}^{1} \mathrm{~d} \xi e^{\mathrm{i} \xi p \cdot z} \phi_{\Pi}(\xi, \mu), \\
&\left.\left\langle 0\left|\bar{\psi}_{F_{1}}(z) \gamma_{\mu} \mathscr{U}(z,-z) \psi_{F_{2}}(-z)\right| V(p, \lambda)\right\rangle\right|_{z^{2}=0}=f_{V} m_{V} p_{\mu} \frac{\varepsilon^{*}(\lambda) \cdot z}{p \cdot z} \int_{-1}^{1} \mathrm{~d} \xi e^{\mathrm{i} \xi p \cdot z} \phi_{V}^{\|}(\xi, \mu) .
\end{aligned}
$$

Here $\xi=2 x-1$ with $x$ and $1-x$ the momentum fractions of the two quarks. The Wilson line $\mathscr{U}$ ensures gauge invariance and the quark flavours $F_{i}$ are chosen to match the pseudo-scalar and vector mesons. All of the DAs $\phi(\xi, \mu)$ are normalised to unity when integrated over $\xi \in[-1,1]$. On the lattice we only access moments of the DAs,

$$
\left\langle\xi^{n}\right\rangle(\mu)=\int \mathrm{d} \xi \xi^{n} \phi(\xi, \mu)
$$

which appear in matrix elements of local operators with $n$ derivatives, see e.g. [2]. We then extract the bare values for $\left\langle\xi^{n}\right\rangle(n=1,2)$ from the lattice using ratios of two-point functions. To give one example, let us consider the second moment of the (longitudinal) vector meson DA, $\left\langle\xi^{2}\right\rangle^{\| \text {,bare }}(\mu)$. 
For large Euclidean times $t$ and $T-t$, the correlation functions give for an appropriate ratio

$$
\begin{aligned}
\frac{\sum_{\vec{x}} e^{\mathrm{i} \vec{p} \vec{x}}\left\langle 0\left|\mathscr{O}_{\{\rho \mu v\}}(\vec{x}, t) V_{\sigma}^{\dagger}(0)\right| 0\right\rangle}{\frac{1}{3} \sum_{i} \sum_{\vec{x}} e^{i \vec{p} \vec{x}}\left\langle 0\left|\mathscr{O}_{i}(\vec{x}, t) V_{i}^{\dagger}(0)\right| 0\right\rangle} \longrightarrow-\mathrm{i}\left\langle\xi^{2}\right\rangle^{\|, \text {bare }} \tanh \left((t-T / 2) E_{V}\right) & \\
\times & \times \frac{1}{3}\left(-g_{\rho \sigma} p_{\mu} p_{v}-g_{\mu \sigma} p_{\rho} p_{v}-g_{v \sigma} p_{\rho} p_{\mu}+\frac{3 p_{\rho} p_{\mu} p_{v} p_{\sigma}}{m_{V}^{2}}\right) .
\end{aligned}
$$

Here $E_{V}$ and $m_{V}$ are the energy and mass of the vector meson with interpolating field $V$. The operator with $n$ derivatives is given by

$$
\mathscr{O}_{\left\{\mu \mu_{1} \ldots \mu_{n}\right\}}(\vec{x}, t)=\bar{\psi}_{F_{1}}(\vec{x}, t) \gamma_{\{\mu} \stackrel{\leftrightarrow}{D}_{\mu_{1}} \ldots \stackrel{\leftrightarrow}{D}_{\left.\mu_{n}\right\}} \psi_{F_{2}}(\vec{x}, t)
$$

where the $\{\ldots\}$ denote symmetrisation of the indices and subtraction of traces. Note that by choosing directions such as e.g. $\mu=\sigma=2, v=4$ and $\rho=1$ one unit of momentum $p_{i} \neq 0$ is enough to extract $\left\langle\xi^{2}\right\rangle^{\| \text {,bare }}(\mu)$ from Eq. (2.4).

The results of fits to ratios like Eq. (2.4) are plotted for both lattice sizes in Fig. 11 Also shown are linear extrapolations of these bare results to the chiral limit along with their error bands. For the lowest moment of the kaon DA such a linear extrapolation in $\left(m_{\mathrm{s}}-m_{\mathrm{q}}\right)$ is predicted by NLO chiral perturbation theory [8]. Note that we have to account for a slightly wrong strange quark mass of $a m_{\mathrm{s}}=0.04$ in our simulation instead of the physical $a m_{\mathrm{s}}=0.0343$ (16) [6]. In case of the lowest moment for $K$ and $K^{*}$, this is done [2] by extrapolating in $\left(m_{\mathrm{s}}-m_{\mathrm{q}}\right)$ where we can simply enter the correct physical quark masses. For the remaining second moments, a correction in $m_{\mathrm{s}}$ could be obtained by comparing the results for mesons with and without strange quarks. However, within errors these differences are small enough to neglect any corrections. Fig. 1] also shows that finite size effects are small and not significant within errors. Finally, the preliminary results for our $\left\langle\xi^{n}\right\rangle(n=1,2)$ at the physical point are given in Tab. 1, along with results by other groups where available. Our values are quoted using perturbative renormalisation to the $\overline{\mathrm{MS}}$ scheme at a scale of $\mu=2 \mathrm{GeV}$. We have already pointed out the clearly observable $S U$ (3)-breaking effects for the kaon DA in [1]. The same now holds for the $K^{*}$.

\begin{tabular}{|c|c|c|c|c|}
\hline & $\left\langle\xi^{1}\right\rangle_{K}$ & $\left\langle\xi^{2}\right\rangle_{K}$ & $\left\langle\xi^{1}\right\rangle_{K^{*}}^{\|}$ & $\left\langle\xi^{2}\right\rangle_{K^{*}}^{\|}$ \\
\hline $24^{3}$ & $0.02893(87)(166)$ & $0.267(11)(13)$ & $0.0342(16)(21)$ & $0.248(17)(12)$ \\
\hline $16^{3}$ & $0.0277(17)(16)$ & $0.282(17)(14)$ & $0.0297(11)(16)$ & $0.255(13)(13)$ \\
\hline [7]+[9] & $0.0272(5)$ & $0.260(6)$ & $0.033(2)(4)$ & \\
\hline & $\left\langle\xi^{2}\right\rangle_{\pi}$ & $\left\langle\xi^{2}\right\rangle_{\rho}^{\|}$ & $\left\langle\xi^{2}\right\rangle_{\phi}^{\|}$ & \\
\hline $24^{3}$ & $0.272(15)(13)$ & $0.237(36)(12)$ & $0.246(10)(12)$ & \\
\hline $16^{3}$ & $0.274(34)(13)$ & $0.245(27)(12)$ & $0.245(11)(12)$ & \\
\hline [9] & $0.269(39)$ & & & \\
\hline
\end{tabular}

Table 1: Our preliminary results at the physical point and both our lattice sizes, in $\overline{\mathrm{MS}}$ at $\mu=2 \mathrm{GeV}$ using perturbative renormalisation. Also included are results from the given references (at $\mu=2 \mathrm{GeV}$ ). 

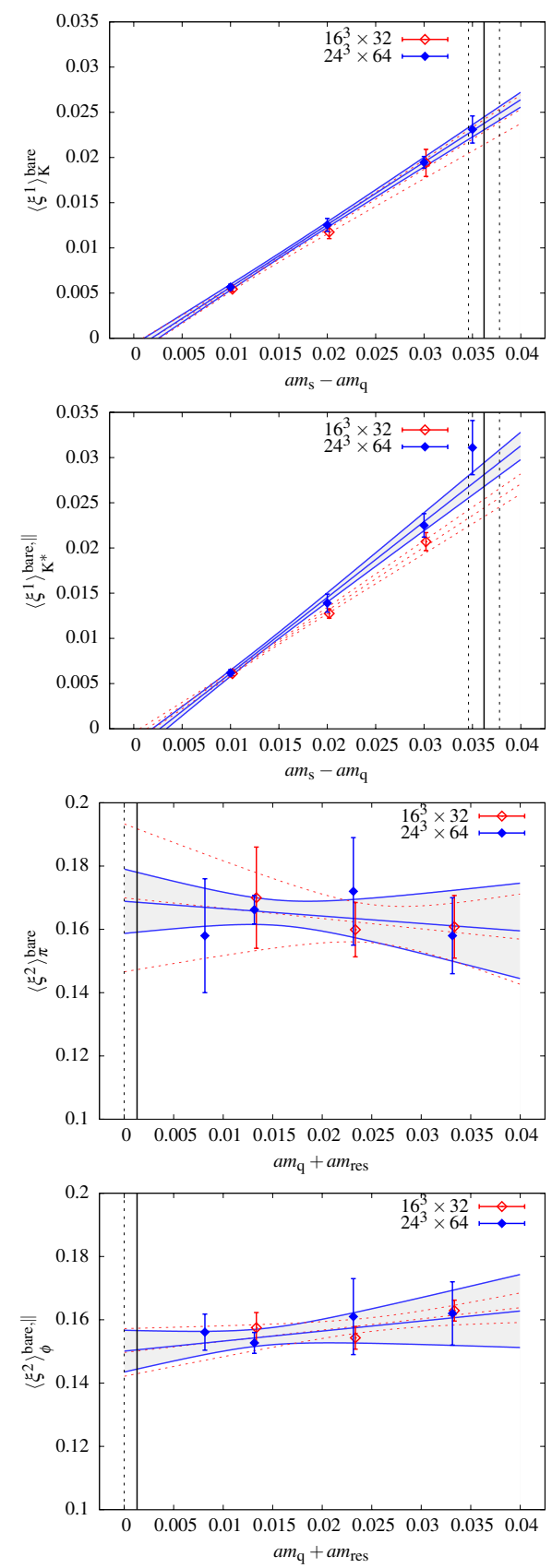
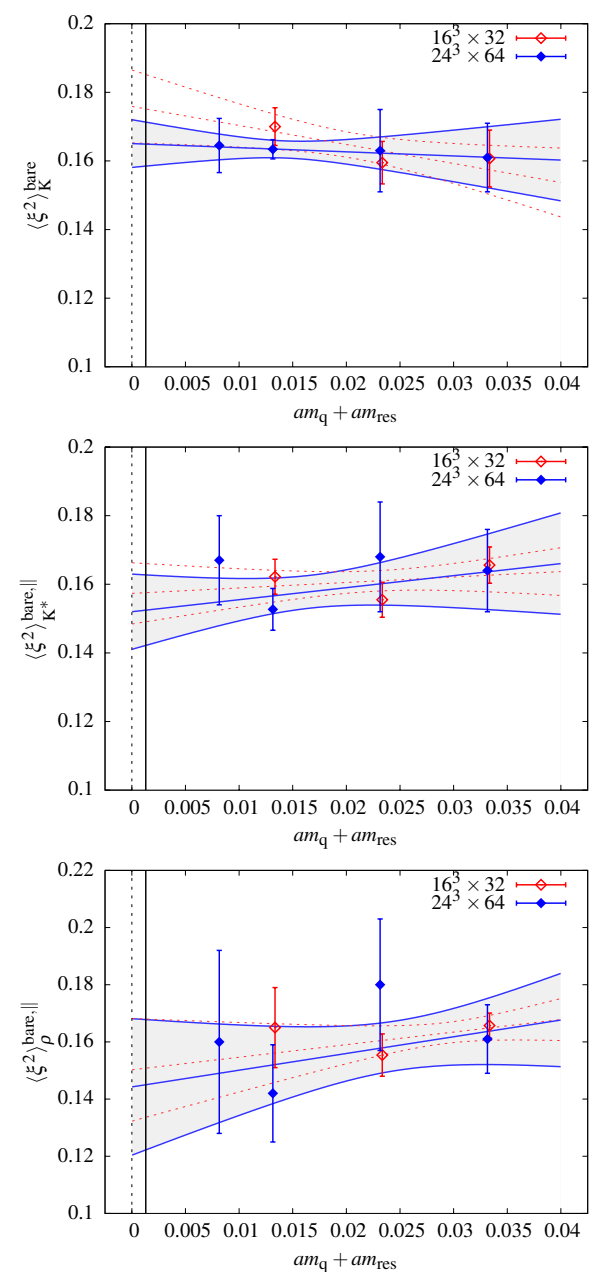

Figure 1: Linear extrapolations of the various moments of the DAs for pseudo-scalar and vector mesons against the mass difference $m_{\mathrm{s}}-m_{\mathrm{q}}$ or the quark mass. Shown are the bare results for $K, K^{*}, \pi, \rho$ and $\phi$ for both lattice sizes. The solid vertical lines mark the physical point with the uncertainty in $m_{\mathrm{s}}$ in case of the lowest moments of $K$ and $K^{*}$.

\section{Non-perturbative renormalisation}

The previous section made use of perturbative renormalisation only. Since this introduces uncertainties due to the known bad convergence of the perturbative expansion, our task now is to compute the necessary renormalisation constants non-perturbatively. For this, we use the RomeSouthampton method which employs a simple renormalisation condition that is useful for any regularisation [3]

$$
\Lambda_{\mathscr{O}}(p)=\left.Z_{\mathscr{O}}(\mu) Z_{q}^{-1} \Lambda_{\mathscr{O}}^{\mathrm{bare}}(p)\right|_{p^{2}=\mu^{2}}=1
$$


Here $\Lambda_{\mathscr{O}}^{(\text {bare) }}(p)$ is the renormalised (bare) vertex amplitude (definition in Eq. (3.3)), $Z_{q}$ is the quark field renormalisation $\left(\psi=Z_{q}^{1 / 2} \psi^{\text {bare }}\right)$ and $Z_{\mathscr{O}}(\mu)$ the desired renormalisation constant $(\mathscr{O}=$ $\left.Z_{\mathscr{O}} \mathscr{O}^{\text {bare }}\right)$ at the scale $\mu$. The renormalisation condition should be applied for scales within an appropriate window [3], $\Lambda_{\mathrm{QCD}} \ll \mu \ll 1 / a$, that we know from our previous calculation [10].

To obtain the bare vertex function, we start by calculating the unamputated Green's function of the operator $\mathscr{O}$ between external off-shell quarks with exceptional momenta, i.e. $p=p^{\prime}$

$$
G_{\mathscr{O}}(p)=\langle\psi(p) \mathscr{O}(0) \bar{\psi}(p)\rangle=\sum_{x}\left\langle\gamma_{5}\left[\sum_{y} S(x, y) e^{\mathrm{i} p y}\right]^{\dagger} \gamma_{5} J_{\Gamma}\left(x, x^{\prime}\right)\left[\sum_{z} S\left(x^{\prime}, z\right) e^{\mathrm{i} p z}\right]\right\rangle .
$$

Where the operator is written as $\mathscr{O}(q)=\sum_{x, x^{\prime}} \exp (\mathrm{i} q x) \bar{\psi}(x) J_{\Gamma}\left(x, x^{\prime}\right) \psi\left(x^{\prime}\right)$ with Dirac structure $\Gamma$ and possible derivatives. We have already written the quark propagators and their Fourier transform in Eq. (3.2) in a way suggesting the use of momentum sources [4]. Instead of using a point source for the inversion, one can perform the inversion on a momentum source $e^{\mathrm{i} p z}$ to find a solution for $S(p)_{x}=\sum_{y} S(x, y) e^{\mathrm{i} p y}$. We then amputate the Green's function and project onto the bare (tree-level) vertex amplitude,

$$
\Pi_{\mathscr{O}}(p)=\langle S(p)\rangle_{G}^{-1}\left\langle G_{\mathscr{O}}(p)\right\rangle_{G}\langle S(p)\rangle_{G}^{-1} \quad \text { and } \quad \Lambda_{\mathscr{O}}(p)=\frac{1}{N} \operatorname{Tr}\left(\Pi_{\mathscr{O}}(p) P_{\mathscr{O}}\right) .
$$

Here the subscript $G$ indicates the gauge average and $N$ ensures the overall normalisation of the trace. The projector $P_{\mathscr{O}}$ has to match the specific Lorentz and kinematical structure of the operator. Examples for quark bilinears without derivatives can be found e.g. in [10]. The operators involving derivatives have a more complicated decomposition that is consistent with their symmetries and tracelessness. Thus more care has to be taken to project onto the correct tree-level contribution, see e.g. [11]. One possibility for an operator like $\bar{\psi} \gamma_{\{\mu} \stackrel{\leftrightarrow}{D}_{v\}} \psi$ would be

$$
\Lambda_{\gamma_{\mu} D_{v}}(p)=\frac{1}{6} \sum_{\substack{\mu, v \\ \mu \leq v}}\left[\frac{\operatorname{Tr}\left[\Pi_{\gamma_{\mu} D_{v}}(p)\left(\gamma_{\mu}+\gamma_{v}\right)\right]}{12\left(\hat{p}^{\mu}+\hat{p}^{v}\right)}-\frac{\sum_{\rho \neq \mu, v} \operatorname{Tr}\left[\Pi_{\gamma_{\mu} D_{v}}(p) \gamma_{\rho}\right]}{12 \sum_{\rho \neq \mu, v} \hat{p}^{\rho}}\right] .
$$

This particular example averages over all possible space-time components of the operator and projector. The symmetrisation is picked up in the first part of the projection, while the second part ensures we pick up the part proportional to the tree-level contribution. We use $\hat{p}_{\mu}=\sin \left(p_{\mu}\right)$ to compensate the kinematic factors.

Since this is an ongoing project, let us only mention a few important findings concerning the advantages of the momentum sources. Fig. 2 compares results from our earlier calculation using point sources, see [10], with the current results. Of course the two calculations agree, the important point is the much smaller statistical error for the momentum sources as shown in the insert of Fig. 2. These results have been obtained from one direction of the momentum for each $(a p)^{2}$ and up to 25 configurations. The point source results on the other hand used 4 separate sources with 75 configurations each and average multiple directions for one value of $(a p)^{2}$, [10]. It is thus possible to reduce the number of inversions while having smaller statistical errors.

The smaller errors make it possible to see lattice discretisation errors which is shown in Figs. 阽 5. Let us consider quark bilinears without derivatives first. Here, one can imagine additional contributions from terms $\propto p^{2}$. Using latticised momenta $\hat{p}$ again and expanding the sine function, 


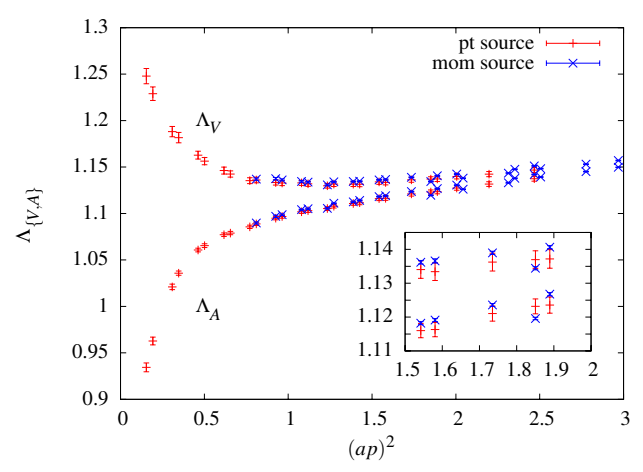

Figure 2: Comparison of the bare vertex amplitude for the vector and axial current from point and momentum sources. Both on $16^{3} \times 32$ lattices for $a m_{\mathrm{q}}=0.03$.

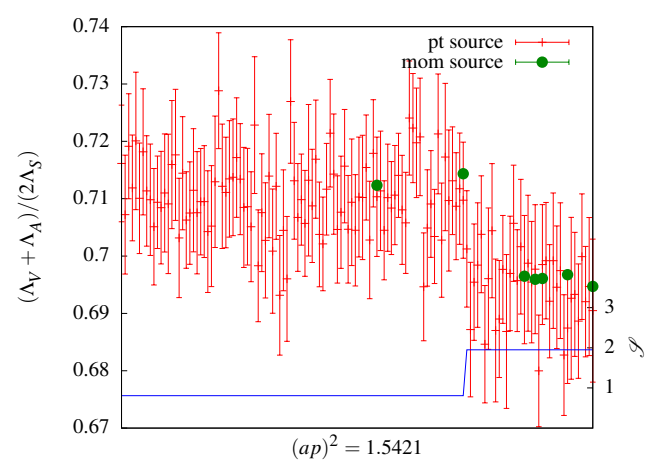

Figure 4: Normalised bare vertex for the two source types, again $16^{3} \times 32, a m_{\mathrm{q}}=0.03$. Only momentum sources reveal different discretisation errors.

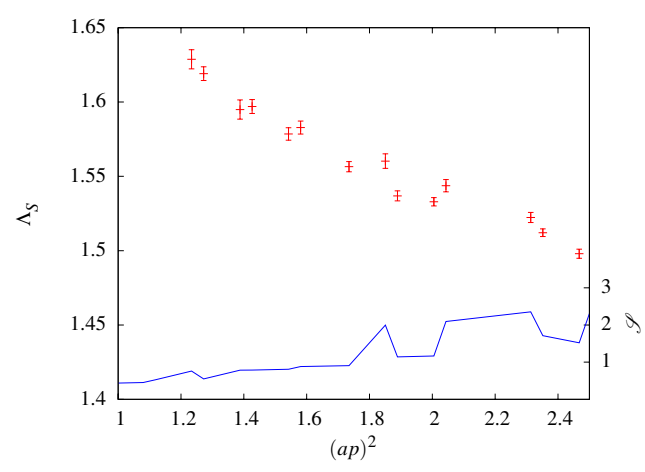

Figure 3: The bare vertex amplitude for the scalar density together with an indicator for discretisation errors, $\mathscr{S}$ (see text). Again $16^{3} \times 32, a m_{\mathrm{q}}=0.03$.

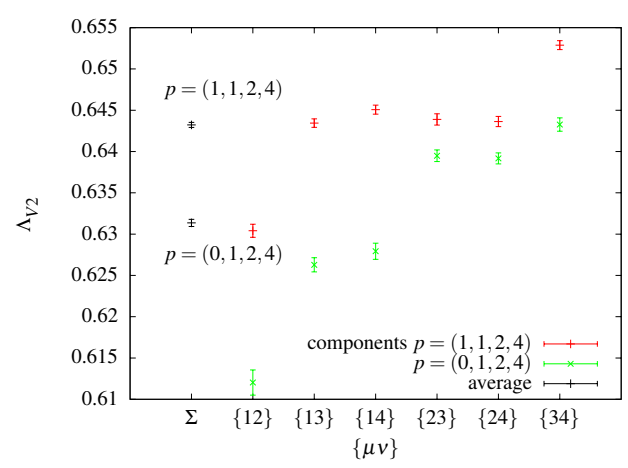

Figure 5: The vertex of the one derivative operator for two momenta, split up into the different choices of Lorentz indices $\{\mu v\}$. Again $16^{3} \times 32$.

we expect possible discretisation errors to be proportional to $\mathscr{S}=\sum_{\mu}\left(2 \pi p_{\mu} / L_{\mu}\right)^{4}$ (taking the leading correction only). In Fig. 目 we plot the bare vertex amplitude of the scalar density along with $\mathscr{S}$. The deviations from the expected smooth behaviour of the vertex is clearly correlated with large changes in $\mathscr{S}$. This becomes even clearer, when we look at a fixed $(a p)^{2}$ but momenta in different directions. The bare vertex should not depend on the latter. However, the different directions result in different values of $\mathscr{S}$.Fig. 7 shows again the scalar density, normalised with the mean vector and axial vector vertex. Included are all directions to one momentum as used in [10], sorted according to the corresponding value for $\mathscr{S}$. The different discretisation errors are only visible for results obtained with momentum sources however. Some examples of these are included in the plot (note that their errors are hidden by the symbols).

Finally, let us mention that the discretisation errors are more difficult for operators with derivatives. Here one can distinguish different directions of the Lorentz indices of the operator w.r.t. the direction of the momentum. This fact is demonstrated for two momenta and the vertex for $\bar{\psi} \gamma_{\{\mu} \overleftrightarrow{D}_{v\}} \psi$ in Fig. 5 . In the continuum all directions are equivalent, on the lattice only choices which have similar momentum components agree within errors, e.g. the combinations $\{\mu v\}=$ $\{23\},\{24\}$. 


\section{Summary}

We have presented an update on our (preliminary) results for distribution amplitudes which have been extended to more pseudo-scalar and vector mesons. The $S U(3)$-breaking effects already found for the $K$ have been confirmed for the $K^{*}$. We did not see a clear sign of finite size effects.

We have also presented our first findings using momentum sources for a non-perturbative renormalisation of our lattice results. Here we see a clear advantage in reducing the statistical errors making it possible to better control the effects due to the lattice discretisation.

\section{Acknowledgements}

We thank our colleagues in RBC and UKQCD within whose programme this calculation was performed. We thank the QCDOC design team for developing the QCDOC machine and its software. This development and the computers used in this calculation were funded by the U.S.DOE grant DE-FG02-92ER40699, PPARC JIF grant PPA/J/S/1998/0075620 and by RIKEN. This work was supported by DOE grant DE-FG02-92ER40699, PPARC grants PPA/G/O/2002/00465 and PP/D000238/1, STFC Grant PP/D000211/1 and from EU contract MRTN-CT-2006-035482 (Flavianet). We thank the University of Edinburgh, PPARC, RIKEN, BNL and the U.S. DOE for providing the QCDOC facilities used in this calculation.

\section{References}

[1] UKQCD Collaboration, P. Boyle et. al., A lattice computation of the first moment of the kaon's distribution amplitude, Phys. Lett. B641 (2006) 67-74 [hep-lat/0607018].

[2] UKQCD and RBC Collaboration, M. Donnellan et. al., Lattice Results for Vector Meson Couplings and Parton Distribution Amplitudes, BoS (LAT2007) 369, [arXiv:0710.0869 [hep-lat]].

[3] G. Martinelli et. al., A general method for nonperturbative renormalization of lattice operators, Nucl. Phys. B445 (1995) 81-108 [hep-lat/9411010].

[4] QCDSF Collaboration, M. Göckeler et. al., Non-perturbative renormalisation of composite operators in lattice QCD, Nucl. Phys. B544 (1999) 699-733 hep-lat/9807044.

[5] RBC and UKQCD Collaboration, C. Allton et. al., $2+1$ flavor domain wall QCD on a $(2 \mathrm{fm})^{3}$ lattice: light meson spectroscopy with $L_{s}=16$, Phys. Rev. D76 (2007) 014504 [hep-lat/ 0701013 .

[6] RBC and UKQCD Collaboration, C. Allton et. al., Physical Results from 2+1 Flavor Domain Wall $Q C D$ and $S U$ (2) Chiral Perturbation Theory, arXiv:0804.0473 [hep-lat].

[7] QCDSF and UKQCD Collaboration, V. Braun et. al., Distribution Amplitudes of Vector Mesons, POS (LAT2007) 144, arXiv:0711.2174 [hep-lat].

[8] J.-W. Chen and I. W. Stewart, Model independent results for SU(3) violation in light- cone distribution functions, Phys. Rev. Lett. 92 (2004) 202001 [hep-ph/0311285.

[9] QCDSF and UKQCD Collaboration, V. Braun et. al., Moments of pseudoscalar meson distribution amplitudes from the lattice, Phys. Rev. D74 (2006) 074501 [hep-lat/0606012].

[10] RBC and UKQCD Collaboration, Y. Aoki et. al., Non-perturbative renormalization of quark bilinear operators and $B_{K}$ using domain wall fermions, arXiv:0712.1061 [hep-lat].

[11] J. A. Gracey, Three loop anomalous dimension of the second moment of the transversity operator in the $\overline{M S}$ and RI' schemes, Nucl. Phys. B667 (2003) 242-260 [hep-ph/0306163]. 